\section{Cambio Psicoterapéutico en niños, niñas y adolescentes que han sido víctimas de agresiones sexuales: Evaluación de la continuidad y} caracterización del cambio posterior a la psicoterapia

Psychotherapeutic change in children and adolescents who have been victims of sexual abuse: Evaluation of the continuity and characterization of change after psychotherapy

\author{
Claudia Capella' \\ (iD) 0000-0002-7252-6605 \\ ccapella@u.uchile.cl
}

Sofía Albornoz

(iD) 0000-0002-1283-5529

sofia.albornoz@ug.uchile.cl

\section{Mariela Vega'}

(iD) 0000-0002-4968-8665

peritomarielavegaegmail.com

\author{
Estrella Azocar \\ (iD) 0000-0003-2845-2220 \\ estrellaazocaregmail.com
Diego Pitrón
(iD) 0000-0002-2609-7387
diego.pitron@ug.uchile.cl

\author{
Camila Gómez \\ iD 0000-0002-4817-9236 \\ camigomez25@gmail.com \\ Rocío Pizarro \\ (iD) 0000-0002-1256-8419 \\ rocio.pizarro@ug.uchile.cl \\ Loreto Rodríguez \\ 0000-0002-4988-0147 \\ loretofrodriguez@gmail.com
}

1 Universidad de Chile. Departamento de Psicología, Santiago, Chile.

Resumen:

Antecedentes: Son escasos los estudios sobre psicoterapia en agresión sexual infantil que incluyan un seguimiento tras su finalización. Objetivo: Explorar la continuidad del cambio psicoterapéutico en niños que han sido víctimas de agresiones sexuales luego de finalizada la psicoterapia, y caracterizarlo, integrando sus perspectivas y la de sus adultos responsables. Método: Se realiza un estudio con metodología mixta, incorporando 10 niños/as y adolescentes entre 7 y 17 años. Se evaluó sintomatología al inicio y final de la psicoterapia y luego de 1 a 2 años que ésta había finalizado. En el seguimiento, se entrevistaron a los niños/as y adolescentes y a sus adultos responsables. Los datos cuantitativos (instrumentos sintomatológicos) se analizaron con pruebas no paramétricas y los cualitativos (entrevistas) mediante análisis narrativo. Resultados: Se aprecian diferencias significativas entre la sintomatología pre, post-terapia y seguimiento evaluada cuantitativamente, mostrando que existen cambios al finalizar la terapia, los que se mantienen en el seguimiento. En las entrevistas se identifican diferentes esferas de cambios logrados durante la terapia que se mantienen posterior a su finalización, destacándose factores favorecedores. Conclusiones: Se discuten elementos relevantes para la mantención del cambio post-terapia, tales como el trabajo con el adulto responsable y el cese del contacto con el agresor.
\end{abstract}

Palabras clave: seguimiento; abuso sexual; psicoterapia; infancia.

\title{
Abstract:
}

Background: There are few studies in psychotherapy in child sexual abuse that includes follow-up after therapy. Objetive: To explore the continuity of psychotherapeutic change in children that have been victims of sexual abuse after therapy ended and to characterize it, integrating their perspectives and the one of their responsible adults. Methods: a study with mix methods was done, incorporating 10 children and adolescents between 7 and 17 years old. Symptomatology was assessed at the beginning and end of psychotherapy, and after 1 to 2 years after therapy ended. At follow up children and adolescents, and their responsible adults were interviewed. Quantitative data (symptomatology instruments) was analyzed with non-parametric tests and qualitative data (interviews) with narrative analysis. Results: Significant differences were found between symptomatology evaluated quantitatively at pre, post-therapy and follow up, showing changes after therapy that maintains at follow-up. With interviews different areas of change accomplished during therapy are identified, which maintains after therapy, highlighting factors that facilitate it. Conclusions: Relevant factors for the maintenance of change after therapy are discussed, such as working with the responsible adult and ending contact with the aggressor.

Keywords: follow-up; sexual abuse; psychotherapy; childhood. 


\section{Introducción}

Las agresiones sexuales infanto-juveniles son consideradas una problemática significativa a nivel social, que tiene una alta prevalencia. A nivel global, Barth et al. (2013) describen una prevalencia de entre 8 a $31 \%$ para mujeres y entre 3 y $17 \%$ para hombres; mientras que en Chile, un reciente estudio mostró que un $26,4 \%$ de adolescentes entre 12 y 18 años reporta haber vivido algún tipo de victimización sexual a lo largo de sus vidas (Pinto-Cortez y Guerra, 2019).

La alta prevalencia adquiere relevancia también en términos clínicos, ya que son variados los estudios que demuestran las consecuencias negativas a corto y largo plazo que esta experiencia trae a quienes han sido víctimas, en distintas esferas (Adams et al., 2018; Cutajar et al., 2010; Guha et al., 2019; Pereda, 2010), pudiendo constituirse en una vivencia traumática (Capella, 2017).

Debido a esto, se considera fundamental el tratamiento psicológico para quienes han sido víctimas, existiendo múltiples investigaciones que muestran los diversos cambios positivos que genera en las víctimas su participación en psicoterapia (Hetzel-Riggin, et al., 2007; SánchezMeca et al., 2011).

En cuanto a la psicoterapia especializada, en Chile la principal institución estatal encargada de la oferta de tratamiento para niños/as y adolescentes' que ha sido víctimas es el Servicio Nacional de Menores (SENAME), que estipula como un objetivo central, favorecer la resignificación de la experiencia abusiva (SENAME, 2015), es decir, un cambio en el significado que la víctima le da a la agresión sexual, permitiendo una mayor comprensión de esta experiencia y una mejor adaptación e integración a su continuo vital (Capella y Gutiérrez, 2014).

Considerando que la resignificación de las agresiones sexuales implica un proceso de cambio, Gómez (2016) plantea que el cambio en estos casos es entendido como un proceso de crecimiento global y una transformación en la manera en que se visualizan a ellos mismos, sus relaciones y el entorno, siendo capaces de construir una narrativa personal y relacional más fortalecida y diversa, a partir de la reconceptualización de la experiencia de agresión sexual (Capella et al., 2018).

En esta propuesta se integra el concepto de cambio psicoterapéutico, entendido como un cambio subjetivo, en la manera de interpretar y explicar el mundo, construyendo nuevas teorías subjetivas (Krause, 2011). El cambio psicoterapéutico implica un proceso irregular pero progresivo, con etapas de avance y retroceso (o estancamiento), por lo que no es un proceso lineal (Altimir et al., 2010; Alves et al., 2014). A su vez, comienza antes de la terapia y continúa después que ésta ha terminado (Krause, 2011).

\footnotetext{
'Se referirá de aquí en adelante como niños, en referencia a niños, niñas y adolescentes, salvo que se haga explícita alguna diferencia etarea o de género.
} 
Las investigaciones sobre cambio psicoterapéutico en agresiones sexuales infanto-juveniles son escasas, más aún los estudios que evalúan cambios de forma integral e incorporando la perspectiva de niños (Capella et al., 2016, 2018; Foster y Hagedorn, 2014; Jessiman et al., 2017). En éstos, los niños mayormente describen cambios positivos en diversas áreas de su vida (autoestima, social, académica), así como una diminución de los efectos negativos asociados a la agresión, teniendo una mayor tranquilidad y una sensación de que se puede seguir adelante con la vida. En los estudios que incorporan la visión de los padres, ellos también evalúan cambios positivos en sus hijos e incluso en ellos mismos (Capella y Rodríguez, 2018; Dussert et al., 2017; Jessiman et al., 2017).

Dichas investigaciones constituyen un gran aporte a la temática, pero ninguna incorpora una visión del cambio posterior a finalizada la psicoterapia. Es crucial comprender dicho proceso en esta población, considerando que en estudios cualitativos previos los niños que están en terapia destacan que esperan seguir cambiando a futuro (Capella et al., 2018) y el cambio es visto como un proceso que continúa post-terapia (Krause, 2011).

El seguimiento ha sido descrito como la evaluación que se realiza una vez que se formaliza el cierre del proceso psicoterapéutico con el niño y su cuidador/a (Combs et al., 2016). Este permite analizar cómo se mantienen o modifican los cambios observados en terapia, detectar cuáles aspectos son visualizados como exitosos o no y facilita una mirada no lineal del proceso de cambio (Combs et al., 2016; Duarte et al., 2017). Los estudios de seguimiento se han focalizado principalmente en población adulta (Duarte et al., 2017; Sembill et al., 2019), predominando metodologías cuantitativas (Flückinger et al., 2014; Melchior et al., 2016).

En cuanto a población infanto juvenil, los escasos estudios de seguimiento (Comb et al., 2016; Muratori et al., 2003; Weiss et al., 2000), se han focalizado en evaluar los efectos- a corto y/o largo plazo- de tratamientos psicoterapéuticos. Aunque las problemáticas a trabajar y los enfoques terapéuticos son diversos, todos estos estudios concluyen que la psicoterapia resulta efectiva en la remisión de síntomas.

Las investigaciones de seguimiento específicas en niños victimizados sexualmente, son aún más escasas, predominando nuevamente un enfoque cuantitativo de medición sintomática (Deblinger, et al., 2006; Deblinger et al.,1999). Estos estudios muestran que los cambios se mantienen en el tiempo, incluso a 2 años de finalizada la psicoterapia, observando que la terapia centrada en el trauma y la cognitivo conductual son más efectivas en el tiempo (Deblinger et al., 2006).

Es necesario complementar los resultados de estas investigaciones con aquellas que relevan las propias narrativas de los participantes respecto de la mantención o modificación de cambios una vez finalizada la terapia y qué factores influyen en esto.

A su vez, si el cambio psicoterapéutico en estos casos es un proceso que requiere del apoyo del medio, en especial de las figuras parentales y que es un proceso conjunto entre cuidadores e hijos (Capella et al., 2018; Dussert et al., 2017; Gómez, 2016), es primordial incorporar dichas perspectivas. 
Así, el objetivo principal de este trabajo es explorar la continuidad del cambio psicoterapéutico en niños que han sido víctimas de agresiones sexuales luego de finalizada la psicoterapia, y caracterizarlo, integrando sus perspectivas y la de sus adultos responsables.

Este estudio busca integrar una evaluación cuantitativa y cualitativa del cambio posterior a la finalización de la psicoterapia, permitiendo aportar información relevante para las intervenciones en el tema.

\section{Metodología}

\section{Diseño}

El presente estudio es una investigación con diseño mixto donde se incorporaron las perspectivas de niños y sus adultos responsables ${ }^{2}$ una vez finalizada la psicoterapia. Se incorporaron evaluaciones cuantitativas, al aplicar instrumentos de medición de sintomatología de manera longitudinal al inicio de la terapia, al finalizar y luego en el seguimiento. En su parte cualitativa, se realizó una entrevista de seguimiento, en un periodo de entre 1 y dos años luego de finalizada la psicoterapia (Hernández et al., 2014).

\section{Participantes}

La muestra del presente proyecto fue seleccionada de manera intencionada, a partir de un universo mayor de participantes pertenecientes a una investigación previa de la misma línea, donde se buscaba caracterizar el contenido y las fases en que se produce el cambio psicoterapéutico en niños que han sido víctimas de agresiones sexuales. Esa investigación incluyó una muestra de 40 niños entre 6 y 18 años que estuvieran en psicoterapia especializada por agresión sexual en Centros colaboradores de SENAME en la Región Metropolitana. Se realizaron entrevistas cualitativas (las cuales no serán incluidas en este artículo) en distintos momentos de la intervención. Además, se aplicaron instrumentos cuantitativos en dos momentos del proceso psicoterapéutico- al inicio de la terapia y al finalizar- con el objetivo de evaluar sintomatología (Capella y Rodriguez, 2018). Luego, en una segunda investigación, se realizó seguimiento a 10 de estos casos luego de 1 a 2 años de finalizada la psicoterapia, abordando en esta investigación el análisis de estos 10 casos.

Estos 10 casos fueron seleccionados para el seguimiento, dentro de los casos de la investigación anterior que hubieran finalizado de manera exitosa su psicoterapia hace 1 año 0 más, utilizando un muestreo intencionado por conveniencia (Hernández et al., 2014; Otzen y Manterola, 2017). Esto, con el objetivo de prever que el re-contactar a los niños y sus cuidadores no significara un retroceso o desestabilización, asociado a las consideraciones éticas evaluadas.

La muestra seleccionada para realizar el seguimiento quedó compuesta por 10 casos, 7 mujeres y 3 hombres, de 10 a 20 años al momento del seguimiento, junto con sus cuidadores (6

2De aquí en adelante, nos referiremos indistintamente a adultos responsables o cuidadores/ cuidadoras. 
madres, 2 abuelas), que habían egresado de sus procesos terapéuticos de centros especializados en intervención en agresiones sexuales, sumando un total de 18 participantes, ya que dentro de la muestra había tres hermanos que compartían adulto responsable.

En relación a las características generales de la muestra (ver tabla 1), el tipo de agresión fue en su mayoría agresión sexual intrafamiliar y el tiempo de intervención psicoterapéutica estuvo mayoritariamente entre 16 y 25 meses.

Tabla 1: Caracterización de la muestra.

\begin{tabular}{|c|c|c|c|c|c|c|c|}
\hline $\begin{array}{l}\text { Pseudónimo } \\
\text { niño }\end{array}$ & $\begin{array}{l}\text { Adulto } \\
\text { responsable }\end{array}$ & $\begin{array}{l}\text { Edad niño al } \\
\text { In icio terapia }\end{array}$ & $\begin{array}{l}\text { Edad niño al } \\
\text { Seguimien to }\end{array}$ & Sexo & Relación con el agresor & $\begin{array}{l}\text { Tiempo en } \\
\text { Terapia }\end{array}$ & $\begin{array}{l}\text { Tiempo desde el fin de la } \\
\text { terapia al seguimien to }\end{array}$ \\
\hline Rocio & Madro* & 8 & 12 & Fomenino & Intrafamiliar & 20 meses & 12 meses \\
\hline Jacinta & Madre & 9 & 12 & Femenino & Intrafamiliar & 25 meses & 12 meses \\
\hline Colos to & Madro & 9 & 12 & Fomenino & Intrafamiliar & 20 meses & 15 moses \\
\hline Noemi & Madre* & 10 & 13 & Femenino & Intrafamiliar & 16 meses & 22 meses \\
\hline Amalia & Madre & 12 & 15 & Femenino & Intrafamiliar & 20 meses & 12 meses \\
\hline Viviana & Madre & 14 & 17 & Femenino & Intrafamiliar & 19 meses & 19 meses \\
\hline Trinidad & Abuela matema & 15 & 18 & Femenino & Intrafamiliar & 18 meses & 17 meses \\
\hline Vicente & Madre* & 7 & 10 & Masculino & Intrafamiliar & 18 meses & 12 meses \\
\hline Rafael & Abuela matema & 13 & 16 & Masculino & Conocido & 18 meses & 17 meses \\
\hline Williams & Madre & $\nabla$ & 20 & Masculino & Intrafamiliar & 7 meses & 26 meses \\
\hline
\end{tabular}

Nota: * Corresponde a la misma adulta responsable, madre de los tres hermanos correspondientes.

Los centros participantes se guían para su intervención en las bases técnicas establecidas por SENAME, donde se estipulan plazos de intervención de doce meses, siendo esto prorrogable por un periodo aproximado de 6 meses más. Se establece la finalización de la intervención en relación al cumplimiento de los objetivos según las bases técnicas: interrupción de la situación de vulneración, resignificación de la experiencia abusiva y el fortalecimiento de los recursos del entorno socio-familiar de los niños (SENAME, 2015). La atención brindada está principalmente orientada a un trabajo psicoterapéutico de los psicólogos/as con los niños y de los trabajadores sociales con el adulto responsable que acompaña.

\section{Procedimientos y Técnicas de Recolección de Datos}

Ambos proyectos contaron con la aprobación del comité de ética de la Investigación de la Institución pertinente.

Luego de tener la aprobación de los directivos de los centros especializados participantes, con apoyo de los profesionales del centro se contactó a niños y sus cuidadores que estuvieran iniciando su proceso de intervención en los centros. Se los invitó a participar, y en caso de 
aceptar, se solicitó la firma de consentimientos y asentimientos informados. Inicialmente se solicitó responder cuestionarios de sintomatología al comienzo de la terapia y más adelante al finalizar la psicoterapia. Se empleó el cuestionario SDO (Strenghts and difficulties questionarie) versión para padres y autoreporte de niños, que han sido evaluados psicométricamente en Chile (Brown et al., 2014; Sánchez, 2017). Consta cada uno de 25 ítems, dividido en 5 escalas: síntomas emocionales, problemas de conducta, hiperactividad, problemas con pares y conducta prosocial (Goodman, 1999). Se aplicó además un instrumento que evalúa síntomas de estrés post-traumático, ya que ha sido considerada como una de las psicopatologías más frecuente en esta población (Cutajar et al., 2010). Se utilizó el Child PTSD Symptom Scale (CPSS), escala de autoreporte, validada en Chile, para niños que han sido víctimas de violencia sexual (Bustos et al., 2009), el cual consta de 17 ítems divididas en 3 escalas: reexperimentación, evitación y aumento de la activación.

Para el seguimiento, se contactó a directivos de dichos Centros, para solicitar autorización de retomar contacto con los casos seleccionados y concretar las entrevistas de seguimiento. Debido a la sensibilidad de la temática, se estableció un protocolo de acción en caso de desestabilización de alguno de los participantes, para contener y evaluar la necesidad de reingresar al programa o gestionar otro tipo de intervención.

Finalmente, para el seguimiento se tomó contacto con los adultos responsables donde se les explicó el estudio de seguimiento y su carácter voluntario. Una vez aceptada la participación (niños y adultos), se acordaron las fechas y el lugar idóneo para la realización de las entrevistas. Una vez reunidos, se procedió a explicar el proyecto, respondiendo dudas, para luego realizar la firma del consentimiento y asentimiento.

En el momento del seguimiento se aplicaron los mismos instrumentos aplicados al inicio y final de la terapia, con el objetivo de evaluar los cambios sintomáticos en el seguimiento. Además, se realizaron entrevistas cualitativas por parte de miembros del equipo de investigación. Para las entrevistas, se elaboraron guiones con temáticas relevantes para el estudio: proceso psicoterapéutico, el contenido y proceso del cambio y los factores favorecedores y dificultadores del cambio, durante y posterior a la terapia, así como las proyecciones de los cambios. Se realizaron por separado las entrevistas a los cuidadores y niños y fueron grabadas en audio para su posterior transcripción. Las entrevistas a niños incluyeron preguntas adaptadas a su nivel evolutivo y fueron complementadas con el uso de dibujo, con el objetivo de facilitar la expresión de sus narrativas. Se utilizó una adaptación del dibujo "antes y después de la terapia" (Capella et al, 2015).

En la tabla 2 se resumen los momentos y técnicas de recolección de datos. 
Tabla 2: Momentos y técnicas de recolección de datos

\begin{tabular}{lll}
\hline Inicio de la terapia & Final de la terapia & $\begin{array}{l}\text { Seguimiento (1 a 2 años de } \\
\text { finalizada la terapia) }\end{array}$ \\
\hline SDQ Padres & SDQ Padres & SDQ Padres \\
SDQ auto-reporte & SDQ auto-reporte & SDQ auto-reporte \\
CPSS & CPSS & CPSS \\
& Entrevista y dibujo al niño \\
& Entrevista al adulto responsable \\
\hline
\end{tabular}

\section{Análisis de Datos}

Los datos cuantitativos obtenidos a través de los instrumentos fueron analizados con apoyo del software SPSS V., utilizando Anova de Friedman, que es una prueba no paramétrica para análisis de regresión lineal y en segundo lugar, el test de Wilcoxon con corrección de Bonferroni, en el caso que el análisis de Anova resultara significativo.

En relación a los datos cualitativos, se analizaron las transcripciones de las entrevistas a través del análisis narrativo temático (Riessman, 2008) y los dibujos, a través del análisis narrativo visual, complementando la información obtenida de las entrevistas (Capella et al, 2015; Riessman, 2008). Estos análisis fueron realizados con apoyo del software ATLAS.TI V7.

El proceso de análisis cualitativo estuvo compuesto por tres momentos. En primer lugar, se realizaron análisis intra-caso de cada entrevista, con el objetivo de extraer los contenidos centrales en las narrativas asociadas al seguimiento, organizando la información en unidades temáticas que permitieron la creación de categorías de análisis. Las entrevistas de los niños fueron complementadas con el análisis narrativo visual de sus dibujos. Un segundo momento, consistió en realizar un análisis inter-caso por participantes, analizando por separado las entrevistas de niños y adultos responsables, con el objetivo de identificar las unidades temáticas características de cada grupo. Finalmente se realizó un análisis de cada caso, integrando la narrativa del niño junto con la de su adulto responsable, para poder identificar aquellos elementos característicos del grupo familiar en torno a los cambios en el seguimiento.

Para asegurar la rigurosidad de los análisis y su validez (Cornejo y Salas, 2011), los análisis fueron triangulados por el equipo de investigación, integrando la mirada de diferentes investigadores. 


\section{Resultados}

\section{Resultados Cuantitativos}

Los resultados se exponen en la tabla 3.

En relación al instrumento SDQ de padres, se observaron diferencias significativas $(p=0.01)$ en la escala total, las que pudieron ser identificadas a través del test de Wilcoxon entre el inicio de la terapia y el final y el inicio de la terapia y el seguimiento. No se encontraron diferencias significativas entre el final de la terapia y el seguimiento.

Respecto de las subescalas, se identificaron diferencias significativas en las subescalas "síntomas emocionales" entre el inicio y el final de la terapia y en la escala "problemas con pares" en los momentos del inicio y final de la terapia y el inicio y el momento del seguimiento.

En el SDQ autoreporte aplicado a los niños, no se apreciaron diferencias significativas ( $p>0.05)$ ni en la escala total, ni en ninguna de las subescalas.

Por otro lado, en el CPSS, el cual evalúa síntomas de estrés post-traumático desde el reporte de los niños, se observaron diferencias significativas $(p<0.01)$ en la escala total, entre el inicio de la terapia y el seguimiento. No se encontraron diferencias significativas entre el final de la terapia y el seguimiento. En el CPSS se apreciaron diferencias significativas en las 3 subescalas entre diferentes momentos de la psicoterapia.

Tabla 3: Resultados aplicación instrumentos

\begin{tabular}{l|llll}
\hline Instrumento & $\begin{array}{l}\text { Promedio } \\
\text { Inicio } \\
\text { terapia }(\mathrm{n}=10)\end{array}$ & $\begin{array}{l}\text { Promedio } \\
\text { Final Terapia } \\
(\mathrm{n}=10)\end{array}$ & $\begin{array}{l}\text { Promedio } \\
\text { Seguimiento } \\
(\mathrm{n}=10)\end{array}$ & $\begin{array}{l}\text { Significancia } \\
\text { Anova de } \\
\text { Friedman }\end{array}$ \\
\hline SDQ Padres & 20.7 & 9.9 & 12.7 & 0.01 \\
SDQ auto-reporte & 12.9 & 11.7 & 12.2 & $>0.05$ \\
CPSS & 25.5 & 14.2 & 12.4 & $<0.01$ \\
\hline
\end{tabular}

Al realizar un análisis descriptivo intra-caso, se observa tanto en el SDO de padres, el SDO de autorreporte y el CPSS, que la mayor parte de los casos disminuyen la sintomatología entre el inicio de la terapia y el final de ésta, y entre el final de la terapia y el seguimiento, y en los que aumenta es sólo levemente, manteniéndose mayormente en el rango sin psicopatología.

\section{Resultados Cualitativos}

Los resultados cualitativos se organizan en cuatro ejes temáticos, que se presentan en la tabla 4. 
Si bien, el énfasis está en aspectos visualizados post terapia, se mencionarán brevemente cambios asociados al proceso psicoterapéutico que los entrevistados relatan en las entrevistas de seguimiento, a modo de brindar una comprensión más profunda del cambio y su mantención.

Para facilitar la lectura, se integraron las narrativas de niños y sus cuidadores (haciendo distinciones cuando corresponda). Con el fin de ejemplificar, se incorporaron viñetas o dibujos de los participantes con sus pseudónimos, a modo de resguardar la confidencialidad.

Tabla 4: Unidades temáticas análisis cualitativo.

\begin{tabular}{|c|c|c|}
\hline Unidades temáticas & Temas & Subtemas \\
\hline $\begin{array}{l}\text { Contenido del cambio en } \\
\text { niños }\end{array}$ & $\begin{array}{l}\text { Cambios emocionales y } \\
\text { sociales } \\
\text { Cambios identitarios } \\
\text { Cambios en relación a la } \\
\text { experiencia abusiva } \\
\text { Cambios en relación a nuevas } \\
\text { dificultades }\end{array}$ & \\
\hline $\begin{array}{l}\text { Contenido del cambio en } \\
\text { el adulto responsable }\end{array}$ & $\begin{array}{l}\text { Cambios en la relación entre el } \\
\text { niño y el adulto } \\
\text { Cambios en el rol parental } \\
\text { Cambios en el bienestar } \\
\text { personal }\end{array}$ & \\
\hline \multicolumn{3}{|l|}{$\begin{array}{l}\text { Proceso de cambio en } \\
\text { niños }\end{array}$} \\
\hline \multirow[t]{2}{*}{$\begin{array}{l}\text { Facilitadores y } \\
\text { obstaculizadores del } \\
\text { cambio en niños }\end{array}$} & Facilitadores & $\begin{array}{l}\text { Apoyo del adulto responsable } \\
\text { Esfuerzos personales } \\
\text { Asociados a la terapia } \\
\text { Aspectos sociales }\end{array}$ \\
\hline & Obstaculizadores & \\
\hline
\end{tabular}

\section{Contenido del Cambio en Niños}

Cambios Emocionales y Sociales. Los niños mencionan que luego de la terapia sienten una mayor estabilidad emocional, superando sensaciones de miedo o inseguridad presentadas al inicio o previo a la terapia, así como percepción de mayor bienestar y felicidad en sus vidas.

"Feliz (...) en un buen momento". (Vicente)

"Estoy mucho más optimista, estoy más perseverante". (Amalia) 
De igual manera es posible observar este aspecto en las narrativas visuales de los niños, donde los cambios se ven reflejados en las expresiones faciales de sus dibujos, desde una expresión de malestar/tristeza en sus rostros al principio de la terapia, a una de felicidad (sonrientes) actual.
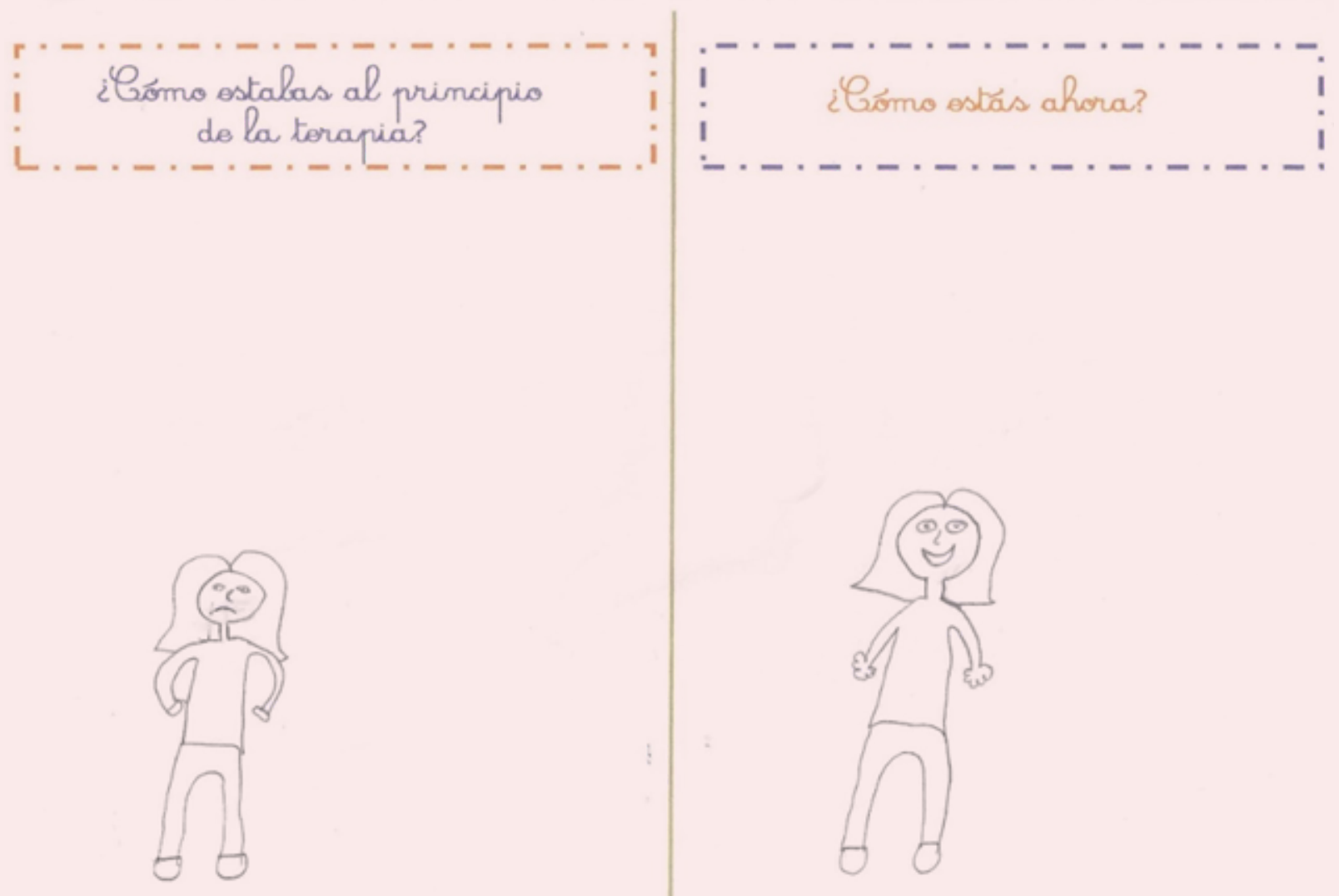

Figura 1. Dibujo de "antes y después" de Celeste

Estos cambios, junto con una mayor expresión emocional, habrían comenzado durante el proceso psicoterapéutico y se han mantenido en el tiempo, permitiéndoles a los niños contar con mayores recursos que favorecen el establecimiento de relaciones interpersonales. Al respecto, todos los entrevistados mencionan una mayor apertura social de los niños y un gradual aumento en la confianza hacia otros pares o la familia, aspecto que connotan como relevante, considerando que previo a la terapia los niños tendían al aislamiento social.

"Ahora me pasa algo y lo digo y es como que no me aguanto nada. Y - nada, ayuda más a - a rodearme de otras personas que solamente estar solo". (Rafael)

Si bien el ámbito social, es descrito tanto por niños como por adolescentes, aparece como un elemento mayormente destacados por los adolescentes, ya que reconocen un mayor interés en establecer relaciones interpersonales. 
De igual manera, los niños tendrían una mayor participación en actividades extracurriculares afines a sus intereses, lo que es visualizado por los entrevistados como un indicador de bienestar.

"Y empezó a ir al gimnasio, iba tres veces a la semana, muy responsable (...) se siente conforme consigo mismo, tiene personalidad". (abuela de Rafael)

Cambios Identitarios. Los niños destacan cambios en su personalidad, visualizando durante y post terapia un mayor empoderamiento respecto de sus vidas y una sensación de sentirse más maduros y capaces.

"Poder llegar a ser lo que anhelas (...) yo anhelo ser un chef destacado y yo sé que puedo serlo, porque no soy de los buenos cocineros y yo sé que puedo ser de los mejores". (Williams)

Esto se visualiza también en algunas de las narrativas visuales, en que los niños se grafican con un mayor tamaño en el momento actual, como el de la figura $N^{0} 1$.

Esto también se traduce en una mayor autonomía y capacidad para resolver situaciones y en la emergencia de metas y anhelos personales, donde prima una actitud activa para focalizarse y esforzarse en el cumplimiento de dichas metas, lo cual también es descrito por sus cuidadoras.

"Era como que leseaba tanto, ahora leseo menos, estoy como más enfocada en no repetir el año (...) ahora trabajo y el colegio no más". (Viviana)

A su vez, las cuidadoras visualizan una mayor confianza y autoestima en sus hijos, en la capacidad para afrontar la vida y defenderse de los obstáculos que puedan venir.

"Se defiende bastante bien (...) ella no se apreciaba nada poh. Era, era un pollito, escondidita, pero ahora no, ahora la veo distinta". (madre de Trinidad).

Cambios en Relación a la Experiencia Abusiva. Todos los entrevistados refieren que durante la psicoterapia y posterior a ésta, visualizan un cambio en relación a tener menos presente la experiencia abusiva.

"Ahora ya eso es como olvidado, que ya no se me viene casi nada a la mente de eso, ya pasé por ese momento triste". (Vicente)

En relación a la experiencia emocional asociada a la agresión, la gran mayoría de los entrevistados aprecian la mantención de cambios que emergieron durante la terapia, recordando la experiencia abusiva con menos dolor o miedo y destacando el sentido de bienestar presente. Para algunos, esto surge a partir de la superación de dicha vivencia, percibiéndola como una experiencia más dentro de otras, que no define la identidad, mientras que en otros, es una experiencia de aprendizaje para el futuro. 
"Cuando ella se dio cuenta de que no era su culpa pudo empezar a como a sanar esto (...) ella se siente sana, dice que ella tiene como una cicatriz (...) pero que hoy día no siente vergüenza por esa cicatriz, es su fortaleza". (Mamá Noemí)

De igual manera, algunas narrativas visuales dan cuenta de cambios elaborativos en relación a la experiencia abusiva, pudiendo vivirla con menos dolor o culpabilidad.

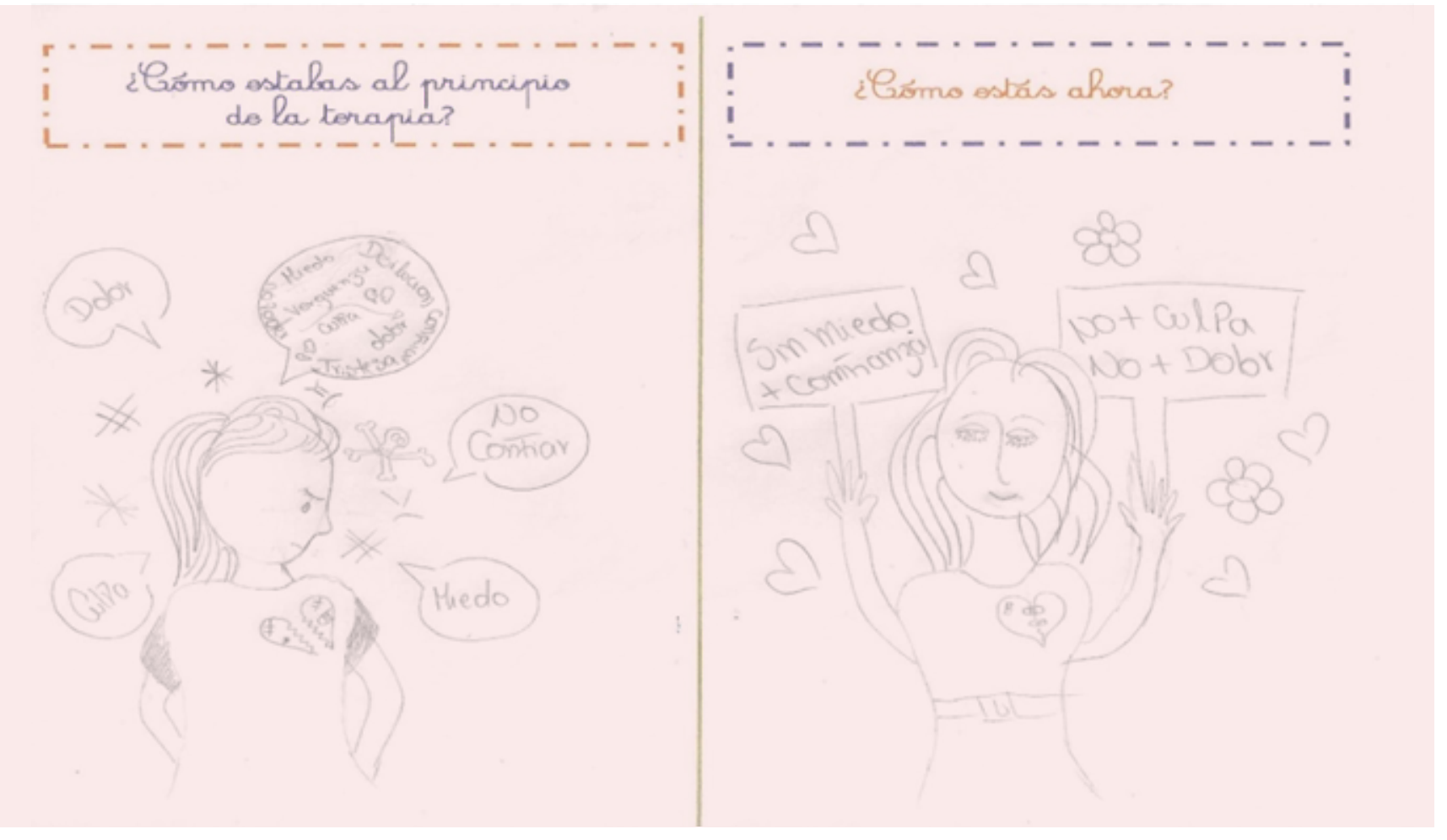

Figura 2: Dibujo "antes y después" de Noemí

Cambios en Relación a Nuevas Dificultades. Preponderantemente desde las narrativas de niños aparecen algunas dificultades posteriores a la finalización de la terapia asociadas a nuevos estresores.

Dos entrevistadas hacen referencias a nuevas dificultades en el aspecto emocional tales como sentimientos de tristeza, aparición de miedos y angustias, así como dificultades para expresar sus necesidades emocionales y un aumento en el nivel de alerta frente a otros, existiendo una mayor desconfianza hacia ellos.

"Desconfío mucho (...) por ejemplo cuando vamos a un negocio (...) desconfío de ellos, porque no sé si son gente buena o gente mala". (Jacinta)

Este malestar y ambivalencia también se observa en las narrativas visuales, donde una de las entrevistadas menciona cambios en su expresión facial respecto del antes y después (antes sentimientos de tristeza y ahora felicidad), pero que la tristeza aparece durante las noches (y en el dibujo graficado en el espejo). 


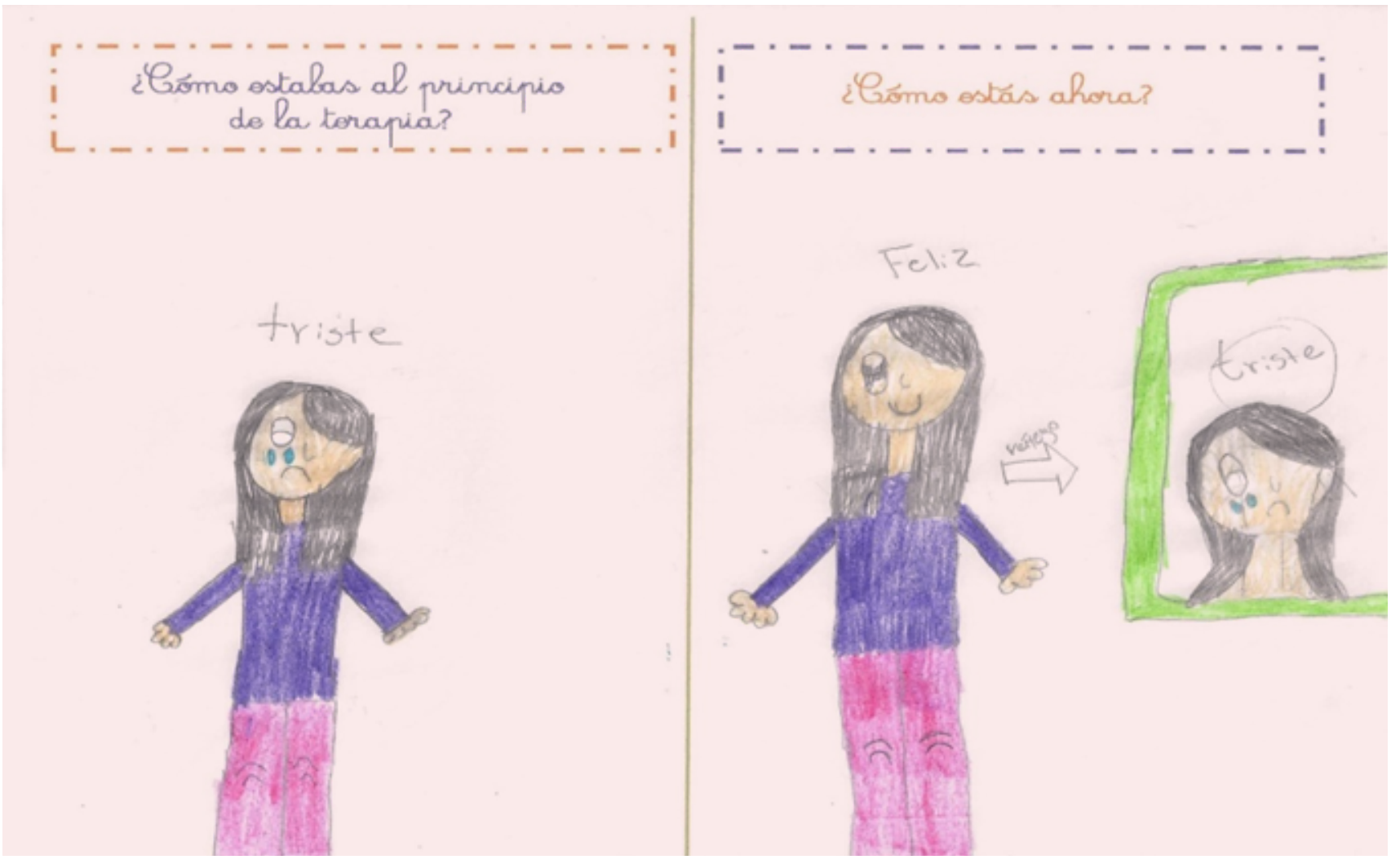

Figura 3: Dibujo "antes y después" de Rocío

Por otro lado, se identifican nuevas dificultades en relación al grupo familiar o a adultos responsables que no participaron de la psicoterapia. Al respecto, algunos niños visualizan cambios negativos en alguna de sus figuras parentales, respecto a los tratos hacia ellos, escasa comprensión de necesidades o pocas redes que les den apoyo por dichas situaciones 3 .

"Yo le he dicho muchas veces a mi mamá que yo necesito volver [a terapia] porque lo quiero terminar y ella me dice que no, que yo estoy bien (...), pero a veces ella no me comprende lo que yo siento". (Rocío)

\section{Contenido del Cambio en el Adulto Responsable}

Cambios en la Relación Entre Niño y Adulto. Tanto durante como post terapia, los entrevistados evalúan un cambio hacia un vínculo de mayor afecto y cercanía, visualizando a la cuidadora como más contenedora y amable que previo a la terapia.

"[E: ¿en qué lo notas tú?], en que es como que antes como llegaba cansada (...) ahora es como que tiene más tiempo para mí y es como mucho más simpática". (Celeste)

3En uno de estos casos (Rocío), se contactó al centro donde se recibía atención para evaluar la situación, realizándose intervenciones con la familia y considerándose el reingreso a tratamiento. 
A través de este vínculo, se construye una relación de mayor confianza, donde por una parte los niños pueden expresar con mayor facilidad sus necesidades e intereses, mientras que en la cuidadora existe una mayor comprensión y empatía con estas necesidades.

"No está tan cerrada en ella (...) trata de ver varios puntos de vista y que también de escucharme en lo que yo pienso, también, o sea ver si ella está errónea o no". (Amalia)

De manera excepcional, dos participantes señalaron cambios que connotan como negativos, por ejemplo, asociado a una relación más aprehensiva o sobreprotectora de parte de la adulta.

Cambios en el Rol Parental. Las cuidadoras destacan el fortalecimiento en su rol parental, cambio visualizado desde la psicoterapia y que se mantiene tras la finalización de ésta. En ese sentido, se releva una mayor capacidad para poner límites y normas que permiten dar estructura a los niños.

"Entonces yo digo 'no Viviana no te quedas hasta muy tarde, diles que tienes que venirte temprano porque yo soy la responsable tuya, bueno ya tienes dieciocho años, pero no quiero que te pase nada', esa es mi preocupación". (abuela de Viviana)

Estrechamente vinculado, las cuidadoras destacan que han desarrollado conductas más protectoras hacia los niños y que eso incluso les ha permitido protegerlos de situaciones de riesgo.

"[A raíz de una situación ocurrida en el colegio] Yo me sentí al principio muy angustiada pero a la vez yo dije 'pucha el me dio la información a tiempo y en seguida pude hacer algo' (...) y esta vez sentí que pude hacer algo, porque fue súper bonito para mi". (Mamá de Vicente)

Cambios en el Bienestar Personal. Un aspecto visualizado tanto durante como post psicoterapia, se vincula a la sensación de mayor bienestar en la vida de las cuidadoras, en comparación a la afectación emocional previo a la psicoterapia. Así, señalan sentirse más satisfechas con sus vidas, más seguras de sus proyectos familiares y/o laborables, lo que a su vez incide en el bienestar de los niños.

"Yo rejuvenecí y mi hija me ve feliz". (madre de Trinidad)

En algunos casos, el espacio de intervención de los niños también significó para ellas una superación del dolor o afectación emocional por la agresión ocurrida e incluso una disminución de la culpabilización por ésta, aspectos que también se mantendrían en la actualidad. 
"Yo encuentro que para las dos, porque a mí me ayudó harto (...) a no tener tanta rabia o qué sé yo, no pensar tanto, porque yo tenía como depresión y eso me ayudó harto". (abuela de Viviana)

\section{Proceso de Cambio en Niños}

La mayoría de los entrevistados describe que los cambios vividos durante la terapia y posterior a ésta son graduales y requieren tiempo, visualizando incluso que algunos cambios se asientan o progresan tras la finalización de la psicoterapia. En algunos casos, también destacan que no visualizaron retroceso o estancamientos.

"Yo creo que el lazo con mi mamá se ha mantenido y ha mejorado un poco". (Williams)

"No, fue avanzando, siempre fue avanzando (...) ese momento del juicio yo pensé que ibamos a tirar para atrás, pero yo estaba muy 'tenemos que llegar a esto, tenemos que hacerlo"'. (madre de Trinidad)

Los entrevistados señalan que son capaces de proyectar a futuro los cambios visualizados en la actualidad, ya sea manteniendo los cambios o generando nuevos. En ese sentido, refieren que podrán desplegar acciones para alcanzar metas y sueños -proyectándose en relación al ámbito laboral y académico- así como proyectar logros de la terapia en sus relaciones o a sus familias.

"Entre broma y broma el Williams me dice que él después se va a ir a vivir solo, yo le digo que sí sé (...) él tiene sus proyectos, es terminar de estudiar y trabajar y él quiere ser un gran chef". (madre de Williams)

Aunque la mayoría de los entrevistados refieren que los cambios se mantienen o progresan, dos niñas también identifican retrocesos en sus procesos de cambios, relacionados a nuevas dificultades post terapia, descritas previamente.

"[E: ¿y cómo es eso que saliste peor?], porque ya pasaron los días y estaba bien todo, pero después como que se empezó a bajar todo (...), peleaba más con mi papá (...), antes peleaba con mi mamá y ahora peleo con mi papá y no debería ser asi". (Rocío)

\section{Facilitadores y Obstaculizadores del Cambio en Niños}

Facilitadores. Corresponden a aquellos elementos que los entrevistados mencionan les ayudaron a realizar los cambios durante la psicoterapia y mantenerlos una vez finalizada.

Apoyo del Adulto Responsable. La mayor comprensión y apoyo de las cuidadoras es visualizado como un facilitador en la mantención de cambios post-terapia en los niños, ya que la mayor disponibilidad, empatía y apoyo de las adultas, les permite a los niños expresar sus necesidades y sentirse acogidos. En algunos casos, el apoyo familiar también fue fundamental 
Cambio Psicoterapéutico en niños, niñas y adolescentes que han sido víctimas de agresiones sexuales...

en este proceso.

"Siento que ella [mamá] siempre ha sido mi pilar fundamental y siento que estoy tirando para arriba, siento que estoy floreciendo, de nuevo". (Williams)

Las cuidadoras destacan como favorecedor en la mantención de los cambios, estar atentas al periodo evolutivo de los niños y de los intereses o cambios que puedan experimentar, brindando apoyo y compañía ante sus diversas inquietudes.

"En algunos temas estoy yo detrás y le digo 'no hija, si tú eres capaz, capaz de eso y mucho más', pero esas herramientas a mí me las enseñaron en la terapia (...) ahora es 'tú puedes (...) aquí estoy para apoyarte'". (Madre de Celeste)

Esfuerzos personales. Todos los entrevistados destacaron el esfuerzo personal y la motivación de los niños para afrontar situaciones difíciles como un facilitador del cambio durante la terapia y para la mantención una vez que ésta finalizó.

"Cuando estoy en un mal momento me acuerdo de las cosas buenas o comienzo a pensar en todo eso y como que salgo adelante, o sea me dan ganas de salir adelante". (Celeste)

Esta voluntad y disposición de los niños resulta fundamental para la prolongación de los cambios, observándose en algunos entrevistados una actitud que tiende a la autorreflexión y al reconocimiento de sus emociones.

"Siento que he sabido ocupar bien mi cabecita (risa) que cuando estoy triste, trato de no tirarme más para abajo (...) cuando estoy triste pensar por qué lo estoy. 0 por qué las cosas van mal". (Amalia)

Asociados a la terapia. Los entrevistados visualizaron aspectos de la intervención y del centro que destacan como facilitadores del cambio en ese momento, en especial el vínculo de confianza y seguridad con el terapeuta, así como la utilización de técnicas lúdicas y/o artísticas afines a sus intereses y necesidades.

"Me apoyaban [terapeutas] mucho en el sentido de lo que yo sentía, ellos siempre juegan conmigo y lo que yo me sentía incómoda ellos no lo hablaban, pero yo dibujaba o pintaba o hacía cualquier otra cosa". (Rocío)

En ese sentido, un facilitador para la mantención de los cambios post terapia se relaciona con el recordar las conversaciones y actividades realizadas en terapia o acordarse del terapeuta y las cosas que les decía.

"[¿Qué ha ayudado a que permanezcan esos cambios?] recordar a la tía [psicóloga]". (Vicente) 
Aspectos Sociales. Otro aspecto destacado especialmente por los niños se relaciona a la dimensión social e interpersonal, destacando que tanto durante como post terapia, el apoyo de pares es relevante para su proceso de cambio.

“(...)todos los fines de semana nos juntábamos [amigas] o salíamos de paseo (...) cuando estoy triste me pregunta mi mejor amiga qué me pasa y empezamos a hablar y es como que no sé, es como que me suelto y ella me entiende". (Celeste)

Obstaculizadores. Corresponden a aquellas situaciones que los entrevistados observan dificultaron el proceso de cambio. Se mencionan menos obstaculizadores que facilitadores, incluso algunos mencionaron que no visualizan obstaculizadores.

Uno de los obstaculizadores más destacados durante el proceso psicoterapéutico corresponde al cambio de terapeuta, en donde la rotación de profesionales en los centros implicó que los niños debían continuar su proceso con un nuevo profesional (o en algunos casos, más de uno) afectando el vínculo y la confianza que se establecía, generando en algunos casos, la sensación de repetir información o retroceso. También, connotan la reiteración de temas o técnicas como obstaculizador de su proceso.

"Con Rocío se produjo una cosa de tener varios psicólogos entre medio, que cambiaban a cada rato, que no había una estabilidad en su terapia, eso la insegurizó más que ayudarla". (Madre de Rocío)

"Como el tema del dibujo, como siempre era lo mismo, que 'dibuja esto, que dibuja los sentimientos que tiene cada parte de tu cuerpo'". (Viviana)

Algunas cuidadoras destacan como obstaculizador la falta de apoyo de familiares significativos o relaciones conflictivas al interior del núcleo familiar.

Posterior a la terapia, en general los entrevistados no reportan obstaculizadores, exceptuando algunos casos donde mencionan el contacto directo o el encuentro con el agresor, así como las situaciones judiciales pendientes, como aspectos dificultadores del cambio.

"Igual obviamente lo sigo viendo a la distancia [agresor], porque aunque pongamos una malla negra y con el sol, igual se ve (...), a veces siento que ellos [familia del agresor] me tienen así como vibra o algo asi'. (Jacinta)

\section{Discusión}

A partir de los datos cuantitativos y cualitativos es posible concluir que se aprecian cambios durante la psicoterapia, y que la mayor parte de éstos se mantiene posterior a la finalización de ésta.

Respecto a los datos cuantitativos, se aprecian cambios desde el reporte de los cuidadores y de los niños. En estos últimos no se aprecian cambios significativos en la evaluación de 
dificultades generales a través del SDQ, ya que en los diferentes momentos reportan escasa sintomatología a través de dicho instrumento, pero si a partir de la evaluación específica de síntomas de estrés post-traumático, lo que podría hacernos pensar que un instrumento que evalúa sintomatología específica pudiera ser más sensible al reporte de los mismos niños. Por otro lado, el SDQ de padres muestra diferencias significativas, tanto entre el inicio y el final de la terapia, como entre el inicio de la terapia y el seguimiento, especialmente en sintomatología emocional y dificultades con pares; mientras que el CPSS entre el inicio de la terapia y el seguimiento y no encontrándose diferencias significativas entre el final de la terapia y el seguimiento. Así, se observan diferentes cambios, hipotetizándose que hay algunos de ellos que toman mayor tiempo en expresarse.

Respecto al contenido del cambio en el seguimiento, apreciado en el análisis cualitativo, la mayoría se desarrolla durante la psicoterapia, pero continúa una vez finalizado el proceso, produciéndose una mantención y/o consolidación de los cambios. Una vez finalizada la terapia, estos cambios continúan desarrollándose e integrándose, siendo referidos por los niños principalmente como un bienestar personal, asociado a cambios a nivel emocional, identitario y social, lo que resulta concordante con lo observado en los instrumentos aplicados durante y post terapia, así como con la literatura existente (Capella et al., 2018; Deblinger et al., 2006).

En relación a la experiencia de agresión, los participantes refieren una disminución significativa en los recuerdos asociados a la experiencia, así como la carga emocional presente, donde se observa una elaboración de la situación traumática, concordante con investigaciones previas (Capella et al., 2016; Capella y Rodriguez, 2018; Draucker et al., 2011) y con la reducción de sintomatología post-traumática observada en la evaluación cuantitativa.

Estos cambios descritos una vez finalizada la terapia coinciden con los cambios descritos durante la terapia (Capella et al., 2016, 2018), por lo cual pareciera que los cambios comienzan durante el proceso psicoterapéutico, permitiendo construir procesos subjetivos que facilitan la resignificación de la experiencia, los cuales se extienden más allá del proceso de psicoterapia (Draucker et al., 2011; Krause, 2011). Así, los objetivos alcanzados durante la terapia presentan una continuidad que entrega estabilidad emocional y la posibilidad de integrar nuevos significados respecto a la experiencia de agresión.

Respecto al proceso de cambio observado en el seguimiento, se reporta mantención en la mayor parte de los cambios, no obstante, se identifican diferencias entre los casos, que dan cuenta del cambio como un proceso no lineal (Altimir et al., 2010) ni homogéneo: en primer lugar, están aquellos participantes que refieren una mejora durante y después del proceso de psicoterapia, manteniendo, fortaleciendo y/o apareciendo nuevos cambios que favorecen el bienestar presente. Hay algunos casos, en que aun cuando se aprecia una mejora y mantención de los cambios, describen algunas dificultades menores. Sin embargo, se apreciaron dos casos en que si bien identifican un mayor bienestar posterior a la terapia, también identifican la aparición de nuevas dificultades. En estos casos existen elementos contextuales asociados a dificultades con los cuidadores o posiblemente un nuevo contacto con el agresor. 
Esto refleja cómo las trayectorias de cambio pueden ser diferentes entre los casos (Davies et al., 2020) y la relevancia de seguir estudiando este proceso. En ese sentido, a raíz de los resultados de la investigación se aprecian elementos relacionados al contexto del niño, tales como la relación con sus cuidadores y familia, así como aspectos personales y de la misma terapia que influyen en el proceso de cambio.

Respecto a los factores personales que favorecen la mantención del cambio, destacan los esfuerzos personales de los mismos niños para poder alcanzar los objetivos propuestos y superar la experiencia de agresión sexual, destacando el rol activo y los recursos de ellos (Capella et al., 2016; Draucker et al., 2011). Por otro lado, entre los factores del proceso terapéutico, se releva la importancia de las intervenciones y el vínculo terapéutico, así como la estabilidad del proceso, generando una mayor confianza aquellos procesos con menos interrupciones y cambios de terapeuta, lo cual tiene importantes implicancias para las intervenciones en estos casos (Capella et al., 2016; 2018; Foster y Hagedorn, 2014; Jessiman et al., 2017).

En cuanto a los elementos contextuales y vinculado a los cambios en los cuidadores, tanto durante como post terapia éstos adquieren un rol fundamental. Los adultos adquieren herramientas que permiten contener y comprender mejor a sus hijos, siendo un factor protector esencial que potenciar, al seguir acompañando el adulto al niño una vez finalizada la terapia. En ese sentido, un cambio que destaca es una relación de mayor confianza y comunicación, coincidiendo con lo observado en la literatura, donde se releva el rol del adulto tanto en el proceso de psicoterapia, como en la mantención de cambios posterior a la psicoterapia (Dussert et al., 2017; Vladimir y Robertson, 2020). Por ende, es imperativo visibilizar el trabajo realizado con estas figuras, ya que las herramientas adquiridas por ellos, repercuten directamente en los cambios que logran y que luego mantienen los niños (Dussert et al., 2017). Similar a otros estudios en que la mayor parte de los cuidadores son figuras femeninas (Dussert et al., 2017; Jessiman et al., 2017), en este estudio solo contamos con la participación de cuidadoras mujeres, por lo cual en futuros estudios sería interesante estudiar este aspecto desde una perspectiva de género.

Finalmente, los participantes refieren las condiciones contextuales como un elemento significativo. Uno de los elementos significados como obstaculizador de la mantención de los cambios es el contacto con el agresor. Un posible contacto con esta figura post-terapia, pudiera generar temor y ansiedad en los niños (Foster y Hagedorn, 2014), por lo que se considera fundamental como implicancia el poder relevar la importancia de las medidas proteccionales que se adoptan una vez finalizada la psicoterapia, ya que si bien el proceso formal termina, para que los niños y sus adultos puedan continuar manteniendo los cambios alcanzados, la interrupción del contacto con el agresor es fundamental.

La presente investigación aporta en el campo de las investigaciones de seguimiento en la temática de las agresiones sexuales, donde la psicoterapia es una herramienta fundamental para lograr elaborarla, siendo relevante poder mirar aquellos elementos de la terapia que generan un impacto más estable y profundo en el tiempo (Capella et al., 2016; Hetzel Riggin et al., 2007). La superación de la experiencia de agresión sexual es un proceso difícil, pero 
que permite dar cuenta de la recuperación y crecimiento a partir de la resignificación de la experiencia traumática y su integración al continuo vital (Capella et al., 2016; Draucker et al., 2011). La combinación del reporte de cambios sintomáticos y subjetivos en este proceso a través de metodología mixta también se considera un aporte, para poder comprender de manera más completa el proceso de cambio.

Como limitación de este estudio, podemos considerarlo inicial debido al escaso número de casos, así como que solo fueron incluidos en el seguimiento participantes que habían finalizado sus procesos de psicoterapia de manera exitosa, por lo cual la mantención de los cambios descrita está asociado a procesos psicoterapéuticos que finalizaron con el alta psicoterapéutica. Lo anterior deja la interrogante sobre el proceso post-terapia en aquellos casos que no logran alcanzar los objetivos terapéuticos planteados, lo cual podría ser considerado en futuras investigaciones, tomando en consideración aspectos éticos involucrados en el seguimiento de estos casos.

\section{Conclusiones y aportes del estudio:}

Se aprecian cambios durante la psicoterapia con niños/as y adolescentes que han sido víctimas de agresiones sexuales, los que mayormente se mantienen en el seguimiento. Los cambios se aprecian en diferentes esferas: sintomáticas, emocionales, sociales, identitarias y vinculadas a la agresión sexual. Se destacan elementos favorecedores para la mantención del cambio post-terapia, como los esfuerzos personales y el apoyo del adulto responsable y obstaculizadores como el contacto con el agresor.

\section{Reconocimientos:}

Se contó con el financiamiento de CONICYT/ANID, a través del proyecto FONDECYT de Iniciación 11140049 "Cambio Psicoterapéutico en Agresiones Sexuales: Perspectiva de los niños/as y adolescentes que han sido víctimas, sus padres y sus psicoterapeutas" y del proyecto FONDECYT Regular 1200627 "Proceso de cambio psicoterapéutico en niños y niñas que han sido víctimas de agresiones sexuales: Hacia un modelo comprensivo de la influencia de factores de los adultos responsables, las intervenciones y la relación terapéutica", así como de la Facultad de Ciencias Sociales de la Universidad de Chile mediante el proyecto de Fortalecimiento de Productividad y Continuidad en Investigación (FPCl) 08-0817 "Trayectorias de Cambio: Perspectiva de niños y adolescentes que han sido agredidos sexualmente y han asistido a terapia, y de sus padres". Se agradece a las instituciones que colaboraron y especialmente a los participantes del estudio por permitirnos acceder a sus experiencias.

\section{Referencias}

Adams, J., Mrug, S. \& Knight, D. (2018). Characteristics of child physical and sexual abuse as predictors of psychopathology. Child abuse \& neglect, 86, 167-177. https://doi.org/gjm67z 
Altimir, C., Krause, M., de la Parra, G., Dagnino, P., Tomicic, A., Valdés, N., Perez, C., Echávarri, O. \& Vilches, O. (2010). Clients', therapists', and observers' agreement on the amount, temporal location, and content of psychotherapeutic change and its relation to outcome. Psychotherapy Research, 20(4), 472-487. https://doi.org/bszzw8

Alves, D., Fernández-Navarro, P., Ribeiro, A., Ribeiro, E. \& Goncalves, M. (2014). Ambivalence and Innovative Moments in Grief Psychotherapy: The Cases of Emily and Rose. Psychotherapy, 51(2), 308-321. https://doi.org/10.1037/a0031151

Barth, J., Bermetz, L., Heim, E., Trelle, S. \& Tonia, T. (2013). The current prevalence of child sexual abuse worldwide: a systematic review and meta-analysis. International Journal of Public Health, 58, 469-483. https://doi.org/10.1007/s00038-012-0426-1

Brown, P., Capella, C. \& Antivilo, A. (2014). Propiedades psicométricas de la versión para padres Strengths and Difficulties Questionnaire. Revista de Psicología, 23(2), 28-44. https://doi.org/10.5354/0719-0581.2014.36146

Bustos, P., Rincón, P., \& Aedo, J. (2009). Validación Preliminar de la Escala Infantil de Síntomas del Trastorno de Estrés Postraumático (Child PTSD Symptom Scale, CPSS) en Niños/as y Adolescentes Víctimas de Violencia Sexual. Psykhe, 18(2), 113-126. https://doi.org/c6ppht

Capella, C. (2017). Desequilibrium and los of the narrative coherence in identity construction: A piagetian perspective on trauma in adolescent victims of sexual abuse. Journal of constructivist psychology, 30(4), 323-328. https://doi.org/hbzt

Capella, C. \& Gutierrez, C. (2014). Psicoterapia con niños/as y adolescentes que han sido víctimas de agresiones sexuales: Sobre la reparación, la resignificación y la superación. Psicoperspectivas, 13(2), 93-105. https://doi.org/chkt

Capella, C., Gutiérrez, C., Rodríguez, L. \& Gómez, C. (2018). Change during pshychotherapy: the perspective of children and adolescents who have been sexualy abused. Research in Psychotherapy: Psychopathology, Process and Outcome, 21(1), 24-39. https://doi.org/hbzv

Capella, C., Lama, X., Rodriguez, L., Águila, D., Beiza, G., Dussert, D. \& Gutierrez, C. (2016). Winning a Race: Narratives of Healing and Psychotherapy in Children and Adolescents Who Have Been Sexually Abused. Journal of child sexual abuse, 25(1), 73-92. https://doi.org/hbzw

Capella, C., \& Rodríguez, L. (2018). Buenas prácticas que favorecen el cambio psicoterapéutico en casos de agresiones sexuales: Integrando la perspectiva de niños/as y adolescentes que han sido víctimas, sus padres y psicoterapeutas. Revista señales, (18), 7-22. https://tinyurl.com/2p9bv54u 
Capella, C., Rodríguez, L., Águila, D., Dussert, D., Lama, X., Gutiérrez, C. \& Beiza, G. (2015). Storied images of psychotherapeutic change: Approaching children's voices through drawings. Research in Psychotherapy: Psychopathology, Process and Outcome, 18(2), 141-151. https://doi.org/10.4081/ripppo.2015.188

Combs, K., Hoag, M., Javorsky, S., \& Roberts, S. (2016). Adolescent Self-Assessment of an Outdoor Behavioral Health Program: Longitudinal Outcomes and Trajectories of Change. Journal of Child and Family Studies, 25(11), 3322-3330. https://doi.org/f893pp

Cornejo, M. \& Salas, N. (2011). Rigor y calidad metodológicos: un reto a la investigación social cualitativa. Psicoperspectivas, 10(2), 12-34. https://doi.org/cs5s

Cutajar, M., Mullen, P., Ogloff, J., Thomas, S., Wells, D., \& Spataro, J. (2010). Psychopathology in a large cohort of sexually abuse children followed up to 43 years. Child Abuse \& Neglect, 34(11), 813-822. https://doi.org/10.1016/j.chiabu.2010.04.004

Davies, S.,Neufeld, S., van Sprang, E., Schweren, L., Keivit, R., Fonagy, P., Dubicka, B., Kelvin, R., Midgley, N., Reynolds, S., Target, M., Wilkinson, P.,van Harmelen, A. \& Goodyer, I. (2020). Trajectories of depression symptom change during and following treatment in adolescents with unipolar major depression. Journal of Child Psychology and Psychiatry, 61(5), 565-574. https://doi.org/10.1111/jcpp.13145

Deblinger, E., Mannarino, A., Cohen, J. \& Steer, R. (2006). A Follow-up Study of a multisite, andomized, controlled trial for children with sexual abuse-related PTSD symptoms. Journal of American Academy of Child and Adolescent Psychiatry, 45(12), 14741484. https://doi.org/btfrhv

Deblinger, E., Steer, R., \& Lippmann, J. (1999). Two-year follow-up study of cognitive behavioral therapy for sexually abused children suffering post-traumatic stress symptoms. Child Abuse \& Neglect, 23(12), 1371-1378. https://doi.org/10.1016/S0145-2134(99)00091-5

Draucker, C.B., Martsolf, D., Roller, C., Knapik, G., Ross, R. \& Stidham, A. (2011). Healing from childhood sexual abuse: a theoretical model. Journal of Child Sexual Abuse, 20(4), 435- 466. https://doi.org/10.1080/10538712.2011.588188

Duarte, J., Fischersworring, M., Martinez, C. \& Tomicic, A. (2017). "I couldn' $t$ change the past; the answer wasn't there": A case study on the subjective construction of psychotherapeutic change of a patient with a borderline personality disorder diagnosis and her therapist. Psychotherapy Research, 29(4), 445-462. https://doi.org/gcz6fd

Dussert, D., Capella, C., Lama, X., Gutiérrez, C., Águila, D., Rodríguez, L. \& Beiza, G. (2017). Narrativas de Padres de Niños, Niñas y Adolescentes que Han Finalizado Psicoterapia por Agresiones Sexuales: Un Proceso de Superación Conjunta. Psykhe, 26(1). https://doi.org/hbz4 
Flückiger, C., Del Re, A. C., Munder, T., Heer, S., \& Wampold, B. E. (2014). Enduring effects of evidence-based psychotherapies in acute depression and anxiety disorders versus treatment as usual at follow-up - A longitudinal metaanalysis. Clinical Psychology Review, 34(5), 367-375. https://doi.org/10.1016/j.cpr.2014.05.001

Foster, J. \& Hagedorn, B. (2014). Through the Eyes of the Wounded: A Narrative Analysis of Children's Sexual Abuse Experiences and Recovery Process. Journal of Child Sexual Abuse 23(5), 538557. https://doi.org/10.1080/10538712.2014.918072

Gómez, C. (2016). Cambio psicoterapéutico en niños, niñas y adolescentes que han sido víctimas de agresiones sexuales: Una aproximación y propuesta teórica [Memoria para optar al título de psicóloga, Universidad de Chile]. https://tinyurl.com/zn3yh5zw

Goodman, R., (1999). The Extended version of the strengths and difficulties Questionnaire as a Guide to child psychiatric Caseness and Consequent Burden. Journal of Child Psychology and Psychiatry, 40(5), 791-799. https://doi.org/10.1111/1469-7610.00494

Guha, A., Luebbers, S., Papalia, N. \& Ogloff, J. (2019). A follow-up study of mental health service utilisation in a cohort of 2433 sexually abused Australian children utilizing five years of medical data. Child abuse \& neglect, 90, 174-184. https://doi.org/hbz7

Hernández, R., Fernández, C. \& Baptista, P. (2014). Metodología de la investigación. McGraw Hill.

Hetzel-Riggin, M., Brausch, A. \& Montgomery, B. (2007). A meta-analytic investigation of therapy modality outcomes for sexually abused children and adolescents: An exploratory study. Child abuse \& neglect, 31(2), 125-141. https://doi.org/10.1016/j.chiabu.2006.10.007

Jessiman, P., Hackett, S. \& Carpenter, J. (2017). Children's and carers' perspectives of a therapeutic intervention for children affected by sexual abuse. Child and Family Social Work, 22(2), 1024-1033. https://doi.org/10.1111/cfs.12322

Krause, M. (2011). Psicoterapia y cambio. Una mirada desde la subjetividad (2a ed.). Ediciones Universidad Católica.

Melchior, H., Schulz, H., Kriston, L., Hergert, A., Hofreuter-Gätgens, K., Bergelt, C., Morfeld, M., Koch, U., \& Watzke, B. (2016). Symptom change trajectories during inpatient psychotherapy in routine care and their associations with long-term outcomes. Psychiatry Research, 238, 228-235. https://doi.org/10.1016/j.psychres.2016.02.046

Muratori, F., Picchi, L., Bruni, G., Patarnello, M., \& Romagnoli, G. (2003). A two-year follow-up of psychodynamic psychotherapy for internalizing disorders in children. American Academy of Child and Adolescent Psychiatry, 42(3), 331-339. https://doi.org/cm452g 
Otzen, T. y Manterola, C. (2017). Técnicas de Muestreo sobre una Población a Estudio. International Journal of Morphology, 35(1), 227-232. https://doi.org/chs5

Pereda, N. (2010). Consecuencias psicológicas a largo plazo del abuso sexual infantil. Papeles del Psicólogo, 31(2), 191-201. https://tinyurl.com/4yeydj4c

Pinto-Cortéz, C. y Guerra, C. (2019). Victimización sexual de niños, niñas y adolescentes chilenos: prevalencia y características asociadas. Revista de psicología, 28(2). https://doi.org/hb2f

Riessman, C. (2008). Narratives Methods for the human sciences. Sage.

Sánchez, V. (2017). Estimación de propiedades psicométricas del Strengths and Difficulties Questionnaire (SDQ) en su versión autoadministrada en población de niños y adolescentes de la ciudad de Santiago de Chile [Memoria para optar al título de Psicóloga, Universidad de Chile]. https://tinyurl.com/2p9dmxjj

Sánchez-Meca, J., Rosa-Alcázar, A. \& López-Soler, C. (2011). The psychological treatment of sexual abuse in children and adolescents: A meta-analysis. International Journal of Clinical and Health psychology, 17(1), 67-93. https://tinyurl.com/4tc3wbcw

Sembill, A., Vocks, S., Kosfelder, J., \& Schöttke, H (2019). The phase model of psychotherapy outcome: Domain-specific trajectories of change in outpatient treatment. Psychotherapy Research, 29(4), 541-552. https://doi.org/gcz6f4

Servicio Nacional de Menores (SENAME) (2015). Orientación Técnica: Línea programas de programas de Protección Especializada en Maltrato y Abuso Sexual Grave (PRM).

Vladimir, M. \& Robertson, D. (2020). The Lived Experiences of Non-Offending Fathers with Children Who Survived Sexual Abuse. Journal of Child Sexual Abuse, 29(3), 312-332. https://doi.org/10.1080/10538712.2019.1620396

Weiss, B., Catron, T., \& Harris., V. (2000). A 2 year follow up of the effectiveness of traditional child Psychotherapy. Journal of Consulting and Clinical Psychology, 48(6), 1094-1101. https://doi.org/10.1037/0022-006X.68.6.1094

\footnotetext{
Para citar en APA

Capella, C., Azocar, E., Gómez, C., Albornoz, S., Pitrón, D., Pizarro, R., Vega, M., \& Rodríguez. L. (2021).

Cambio Psicoterapéutico en niños, niñas y adolescentes que han sido víctimas de agresiones sexuales: Evaluación de la continuidad y caracterización del cambio posterior a la psicoterapia. Terapia Psicológica (En línea), 39(3), 329-352. https://doi.org/10.4067/S0718-48082021000300329
} 